\title{
Group-Based Behavioural Intervention for Smoking Cessation: Is it All in Their Heads?
}

\author{
Mohamad Haniki Nik Mohamed \\ International Islamic University Malaysia
}

Group-based counselling for smoking cessation has been shown to be an effective form of behavioural intervention. A meta-analysis of 50 randomised controlled trials (RCTs) by Mottillo et al. (2009) showed that the odds ratio (OR) for individual counselling (23 RCTs, $n=$ $8646)$ is 1.49 (95\% confidence interval $(\mathrm{CI})=1.08-2.07)$ vs. $1.76(95 \% \mathrm{CI}=1.11-2.93)$ for group counselling (12 RCTs, $n=3600)$.

An earlier meta-analysis of 53 trials by Stead and Lancaster (2005) for the Cochrane Collaboration demonstrated that both individual and group-based behavioural interventions are efficacious in assisting smokers quit. They found an increase in cessation with the use of a group programme $(n=4375$, relative risk $(\mathrm{RR})=1.98,95 \%$ (CI) 1.60 to 2.46$)$ and concluded that it is better for helping people quit smoking compared to self-help and other less intensive interventions. However, there was not enough evidence to evaluate whether groups are more effective or cost-effective than intensive individual counselling. This conclusion remained, subsequent to another review in 2009. Furthermore, the authors noted that there was limited evidence that the addition of group therapy to other forms of treatment, such as advice from a health professional or nicotine replacement, produced extra benefit.

In the studies used for the analyses, group counselling is defined as having a minimum of two group meetings, and follow-up of smoking status at least 6 months after the start of the program, with carbonmonoxide $(\mathrm{CO})$ validated cessation. Most programs used between six and eight sessions, with the first few sessions devoted to discussion of motivation for quitting, health benefits, and strategies for planning a quit attempt. Participants may also keep records of the number of cigarettes smoked and the triggers for smoking (self-monitoring). Part of the group process also includes discussion and sharing of experiences and problems (intra-treatment social support), as well as on ways to seek appropriate support from friends, colleagues and family (extra-treatment social support). A range of other problem-solving skills may also be introduced, including identifying high-risk situations for relapse, generating solutions and discussing or rehears- ing responses. Some programs incorporate more specific components intended to help manage poor mood or depression associated with quitting and withdrawal.

Can we then compare these findings to non-RCTs of group-based behavioural interventions? It has been recently discussed that the use of large-group motivational seminars may also be effective and cost-effective. An observational study of 223 smokers who attended Allen Carr's 6-hour seminar found a quit rate of at least $40 \%$ at 1 -year follow-up. ${ }^{1}$ Following this, Moshammer \& Neuberger (2006) reported an impressive 50\% success rate for long-term abstinence, that is, at 3 years post seminar, for 510 smokers at a steel-plant who received an intensive group counselling in a single session of 6 hours, again based on Allen Carr's Easyway quitting method.

This method operates with the underlying hypothesis that smokers' beliefs about smoking (e.g., smoking helps them to relax, cope with stress or concentrate) create the perceived need or desire to smoke. Hence, trying to quit while maintaining these beliefs leads to a persistent emotional conflict, where part of a smoker wants to quit, but another part still wants to smoke. Furthermore, the withdrawal symptoms reported by those trying to quit (i.e., irritability, anxiety, and so on) are supposedly the physical manifestations of this emotional conflict. Such a seminar operates on the concept of helping smokers to eliminate all of the beliefs they have that created the desire to smoke in the first place. Supporters of such method agree that the only way to remove this conflict is to challenge the beliefs upon which the desire to smoke are based.

Such a hypothesis can be challenged, based on what is currently known about smoking, specifically, nicotine addiction. Newlin (2008) proposed that nicotine addiction is actually psychoneurobiological, not a form of mind-body dualism.

More importantly, can we rely upon findings from the two observational studies? In medicine, this is hardly acceptable. In fact, the high quit-rate reported included effects of follow-up sessions by occupational health personnel at the smokers' worksite. Furthermore, biochemical validation was done randomly in only 61 (12\%) of the 510 respondents. A Cochrane review of 
workplace interventions (Cahill, Moher, \& Lancaster, 2008) found that proven stop-smoking methods, such as group therapy, individual counselling and nicotine replacement therapy, are equally effective when offered in the workplace. There is also the possibility that, as the workplace study enrolled people from one company en masse, there could be some influence on efficacy as a result of this - people might have been more likely to quit because many other smokers around them were doing so at the same time, which would boost the apparent success of that method. These statements are similar to the earlier recommendations by the UK National Institute for Health and Clinical Excellence (NICE) in 2007.

Should we then disregard such methods? Not entirely. Another NICE guidance in 2008 recommended that research commissioners and funders should commission high-quality and, where appropriate, comparative studies to determine the short- and longterm effectiveness of Allen Carr's method of stopping smoking. ${ }^{2}$ This provides the opportunity for more rigorous and scientific studies, that is, RCTs, of such methods, so that more smokers will reap the benefits and ultimately the evidence will speak for itself.

\section{Conflict of Interests}

Appointed as a key opinion panel member of selected pharmaceutical companies, received travel sponsorship for scientific conferences and honoraria for speaking engagement from various pharmaceutical companies, including Pfizer, Johnson \& Johnson and GSK.

\section{Endnotes}

1 Hutter, H.P., Moshammer, H., \& Neuberger, M. (2006). Smoking cessation at the workplace: 1 year success of short seminars. International Archives of Occupational and Environmental Health, 79, 42-48. DOI 10.1007/s00420005-0034-y

2 National Institute for Health and Clinical Excellence (NICE) Public Health Guidance 10. (2008). Smoking cessation services in primary care, pharmacies, local authorities and workplaces, particularly for manual working groups, pregnant women and hard to reach communities. Retrieved May 11, 2011, from www.nice.org.uk/PH010.

\section{References}

Cahill, K., Moher, M., \& Lancaster T. (2008). Workplace interventions for smoking cessation. Cochrane Database of Systematic Reviews, Issue 4. Art. No.: CD003440. DOI: 10.1002/14651858.CD003440.pub3

Moshammer, H., \& Neuberger, M. (2007). Long-term success of short smoking cessation seminars supported by occupational health care. Addictive Behaviors, 32, 1486-1493. DOI:10.1016/j.addbeh.2006.10.002

Mottillo, S., Filion, K.B., Belisle, P., Lawrence, J., Gervais, A., O'Loughlin, J., ... Tremblay, M. (2009). Behavioural interventions for smoking cessation: a meta-analysis of randomized controlled trials. European Heart Journal, 30(6), 718-730. DOI:10.1093/eurheartj/ehn552

National Institute for Health and Clinical Excellence. (2007). Workplace health promotion: How to help employees to stop smoking. Retrieved May 11, 2011, from www.nice.org.uk

Newlin, D.B. (2008). Are 'physiological' and 'psychological' addiction really different? Well, no! ... um, er, yes? Substance Use \& Misuse, 43, 967-971. DOI: 10.1080/ 10826080802097389

Stead, L.F., \& Lancaster, T. (2005). Group behaviour therapy programmes for smoking cessation. Cochrane Database of Systematic Reviews, Issue 2. Art. No.: CD001007. DOI: 10.1002/14651858.CD001007.pub2. (Updated 2009). 